This article was published in Microscopy and Microanalysis, 21, 2015 http://dx.doi.org/10.1017/S1431927615013835

\title{
Highly Ordered Hexagonal Arrays of TiO2 Nanotubes
}

Arlete Apolinário ${ }^{1}$, Célia T. Sousa ${ }^{1}$, João Ventura ${ }^{1}$, José D. Costa ${ }^{1}$, Diana C. Leitão ${ }^{2}$, José M. Moreira ${ }^{1}$, João

B. Sousa, ${ }^{1}$ Luísa Andrade ${ }^{3}$, Adélio M. Mendes ${ }^{3}$ and João P. Araújo ${ }^{1}$

1. IFIMUP and IN, Departamento de Física e Astronomia, Faculdade de Ciências da Universidade do Porto, Rua do Campo Alegre 687, 4169-007 Porto, Portugal

2. INESC-MN and IN, Rua Alves Redol 9, 1000-029 Lisboa, Portugal

3. LEPABE- Dep. Engenharia Química-Faculdade de Engenharia, R. Dr. Roberto Frias, 4200-465 Porto, Portugal

Highly-ordered $\mathrm{TiO}_{2}$ nanotubes (NTs) have gained much importance for application in hydrogen production by water splitting (photoelectrochemical cells) and the dye-sensitized solar cells (DSCs) [1,2]. The $\mathrm{TiO}_{2}$ NTs can be synthesized using a titanium foil in fluoride containing electrolytes via electrochemical anodization method. The NTs geometry depends on different anodizing parameters (electrolyte type and concentration, $\mathrm{pH}$, time, applied potential) that determine the tube features (length, pore diameter, wall thickness, etc.). $\mathrm{TiO}_{2}$ NTs arrays were synthesized by an electrochemical anodization of a Ti foil (two-electrode cell) with an anodization potential of $60 \mathrm{~V}$ for $17 \mathrm{~h}$, in an ethylene glycol solution containing $\mathrm{NH}_{4} \mathrm{~F}(0.3 \mathrm{wt} \%)$ and $\mathrm{H}_{2} \mathrm{O}(2 \mathrm{wt} \%)$ at room temperature [3]. It was implemented, prior to the anodization, three different pre-treatments on the Ti foil: a chemical etching with $4 \% \mathrm{HF}$ solution, a mechanical polishing and an electropolishing in a $\mathrm{H}_{2} \mathrm{SO}_{4} / \mathrm{HF}$ solution, with an applied potential of $10 \mathrm{~V}$ during $4 \mathrm{~min}$. In this work, it is described the impact that a simple pre-treatment has to the template growth and final thickness after a single anodization step, as well as on the template quality (NTs organization and domain size). For this purpose, the topography of the Ti surface (prior to the anodization) with these 3 pre-treatments and an asrolled Ti sample was investigated by Atomic Force Microscopy. Roughness studies were compared with the NTs template thickness and organization quality. Highly self-ordered arrays of $\mathrm{TiO}_{2}$ NTs were obtained, and found that pre-treatments that decrease the Ti surface roughness are a crucial step in the $\mathrm{TiO}_{2} \mathrm{NTs}$ electrochemical anodization syntheses for obtaining: a fast NTs growth attaining a highest final template thickness; an enhancement in the NTs organization quality reaching highly ordered hexagonal NTs arrays in larger areas [3].

References:

[1] B. O’Regan, M.Gratzel, Nature, 353,737-740, (1991).

[2] M. Gratzel, Photoelectrochemicalcells, Nature, 414 (2001) p.338

[3] A. Apolinário et al. Journal of Materials Chemistry A, 2 (24), 9067-9078, 2014. 

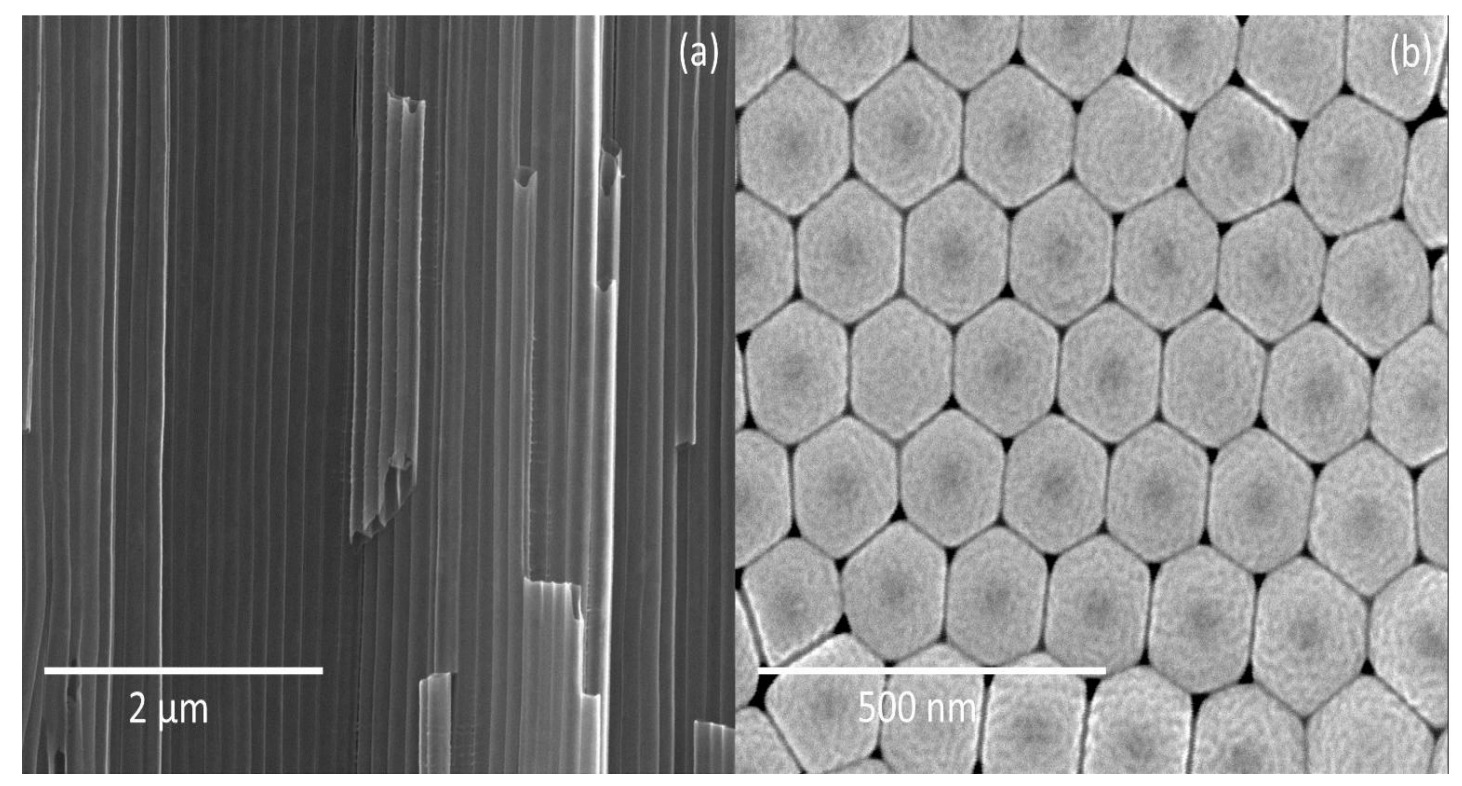

Figure 1. SEM images of $\mathrm{TiO} 2$ NTs with hexagonal arrangement obtained by anodization: (a) cross- section view and (b) bottom view. 